\title{
Laser desorption time-of-flight mass spectrometry of ultraviolet photo-processed ices
}

D. M. Paardekooper, J.-B. Bossa, K. Isokoski, and H. Linnartz

Citation: Review of Scientific Instruments 85, 104501 (2014); doi: 10.1063/1.4896754

View online: http://dx.doi.org/10.1063/1.4896754

View Table of Contents: http://aip.scitation.org/toc/rsi/85/10

Published by the American Institute of Physics

\section{Articles you may be interested in}

Mechanisms for the formation of thymine under astrophysical conditions and implications for the origin of life The Journal of Chemical Physics 144, 144308 (2016); 10.1063/1.4945745

Water formation by surface $\mathrm{O}_{3}$ hydrogenation

The Journal of Chemical Physics 134, 084504 (2011); 10.1063/1.3532087

Surface science investigations of photoprocesses in model interstellar ices Journal of Vacuum Science \& Technology A: Vacuum, Surfaces, and Films 26, 919 (2008); 10.1116/1.2834687

Production and characterization of supersonic carbon cluster beams

The Journal of Chemical Physics 81, 3322 (1998); 10.1063/1.447994

The simulator for icy world interiors: A $700 \mathrm{MPa}$ pressure system for impulsive stimulated scattering and other optical measurements, with thermal control from -20 to $100{ }^{\circ} \mathrm{C}$

Review of Scientific Instruments 79, 105105 (2008); 10.1063/1.3000001

Mechanisms of $\mathrm{H}_{2} \mathrm{O}$ desorption from amorphous solid water by 157-nm irradiation: An experimental and theoretical study

The Journal of Chemical Physics 139, 164702 (2013); 10.1063/1.4825239

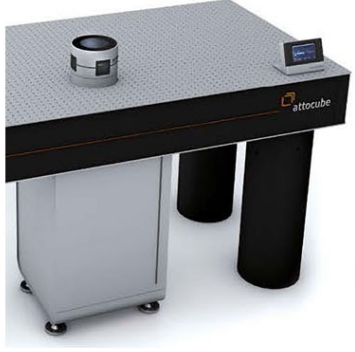

Obstruction free access

optical table with integrated cryocooler

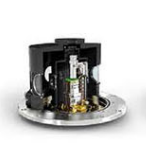

Various 0 bjective 0 ptions attocube

\section{attoDRY800}

- Cryogenic Temperatures

- Ultra-Low Vibration

- Optical Table Included

- Fast Cooldown
$5 \%$ DISCOUNT

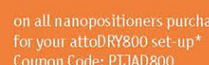




\title{
Laser desorption time-of-flight mass spectrometry of ultraviolet photo-processed ices
}

\author{
D. M. Paardekooper, ${ }^{\text {a) }}$ J.-B. Bossa, K. Isokoski, and H. Linnartz ${ }^{\text {b) }}$ \\ Raymond and Beverly Sackler Laboratory for Astrophysics, Leiden Observatory, Leiden University, \\ PO Box 9513, 2300 RA Leiden, The Netherlands
}

(Received 18 June 2014; accepted 17 September 2014; published online 10 October 2014)

\begin{abstract}
A new ultra-high vacuum experiment is described that allows studying photo-induced chemical processes in interstellar ice analogues. MATRI ${ }^{2}$ CES - a Mass Analytical Tool to study Reactions in Interstellar ICES applies a new concept by combining laser desorption and time-of-flight mass spectrometry with the ultimate goal to characterize in situ and in real time the solid state evolution of organic compounds upon UV photolysis for astronomically relevant ice mixtures and temperatures. The performance of the experimental setup is demonstrated by the kinetic analysis of the different photoproducts of pure methane $\left(\mathrm{CH}_{4}\right)$ ice at $20 \mathrm{~K}$. A quantitative approach provides formation yields of several new species with up to four carbon atoms. Convincing evidence is found for the formation of even larger species. Typical mass resolutions obtained range from $\mathrm{M} / \Delta \mathrm{M} \sim 320$ to $\sim 400$ for $\mathrm{CH}_{4}$ and argon, respectively. Additional tests show that the typical detection limit (in monolayers) is $\leq 0.02$ ML, substantially more sensitive than the regular techniques used to investigate chemical processes in interstellar ices. (C) 2014 AIP Publishing LLC. [http://dx.doi.org/10.1063/1.4896754]
\end{abstract}

\section{INTRODUCTION}

The astronomical flagship facility ALMA (Atacama Large Millimeter Array) has started to produce data with a spectral and spatial resolution that is setting new standards. It is expected that the large number of ions, radicals, small and complex molecular species already observed in the interand circumstellar medium (ISCM) - 180 so far - will increase rapidly. This further highlights the rich chemistry occurring in star and planet forming regions. However, detailed knowledge of the exact formation pathways of many of the observed species is limited. Decades of astronomical observations, laboratory studies and astrochemical modelling point toward a complex interplay between the gas phase and the solid state. ${ }^{1}$ Particularly, many of the saturated species-for example, dimethylether or glycolaldehyde, but also waterare expected to form on icy dust grains, as present in dark interstellar clouds, upon thermal processing, vacuum UV irradiation, atom bombardment, or interaction with cosmic rays or free electrons. ${ }^{2,3}$ Whereas gas phase reactions of astronomical interest have been simulated under fully controlled experimental conditions for decades, this is not the case for reactions taking place in interstellar ice analogues, where separate solid state reactions can be studied in the laboratory roughly since 2000. On the contrary, since tens of years it is known that complex organic material, including amino acids, forms from energetically processing of bulk ices composed of astrochemical relevant constituents upon ultraviolet irradiation ${ }^{4-12}$ and proton irradiation, ${ }^{13,14}$ but the physical-chemical processes at play are not well understood. Moreover, the interpretation of this data is difficult, since the measurements were performed ex situ; residues were heated and exposed to air before analy-

a) dmpaardekooper@strw.leidenuniv.nl

b)linnartz@strw.leidenuniv.nl sis in a gas chromatograph became possible. More recently, in situ analysis of energetically processed ices have focused on the formation of the smallest amino acid glycine. ${ }^{15-17}$ In order to fully understand the complex solid-state astrochemical network responsible for molecular complexity in space, dedicated in situ laboratory experiments are needed, and this is the topic of the present paper. (See also Ref. 18 for a more recent overview.)

The traditionally used analytical methods in solidstate laboratory astrophysics are Fourier Transform InfraRed (FTIR) spectroscopy and Temperature Programmed Desorption (TPD) combined with Quadrupole Mass Spectrometry (QMS). Although sensitive, these techniques are inherently prone to uncertainty. Infrared reflection (RAIRS) or transmission spectroscopy enables remote analysis of the sample composition without chemical alteration. However, depending on the specific ice mixture at hand and the absorption band strength of its constituents the detection limit is $\sim 1 \%$. Molecules without a dipole moment will stay concealed, since they lack IR active modes (e.g., $\mathrm{N}_{2}$ and $\mathrm{O}_{2}$ ). For species with IR active modes, even a qualitative interpretation of the acquired spectra can be limited, due to spectral overlap, specifically as with increasing level of complexity the number of vibrational modes increases. ${ }^{3}$ TPD combined with QMS is more sensitive than FTIR, but comes inherently with the destruction of the ice. Moreover, the increase in temperature may induce thermally activated reactions ${ }^{19}$ and can change ice morphology. ${ }^{20,21}$ For these reasons it is common to combine RAIRS and TPD. ${ }^{3}$

It is clear; to realize progress, technological advances are needed. Indeed, recently a number of new developments have been reported. ${ }^{22-32}$ Successful experiments have been reported in which TPD has been combined with vacuum UV (VUV) single photo-ionisation time-of-flight mass spectrometry (TOF-MS), measuring the mass from desorbing 
species from the ice. ${ }^{22,23}$ Diagnostic techniques such as Substrate/Matrix Assisted Laser Desorption Ionisation Time-ofFlight Mass Spectrometry ((S/M)ALDI-TOF-MS) have been introduced. ${ }^{26,27,31}$ In the case of the MALDI-technique, a laser is used to desorb large molecules by exciting vibrational modes of the matrix molecules in which the molecules of interest are embedded. A similar approach was recently applied, to investigate radiation-induced processes of Polycyclic Aromatic Hydrocarbons (PAHs), embedded in interstellar ice analogous, using Resonant Enhanced Multi Photon Ionisation (REMPI) as ionisation source. ${ }^{24,25}$

Alternatively, the laser energy can be coupled into the substrate to induce the thermal desorption of ice constituents. ${ }^{28,29,33-36}$ The desorbed ice constituents are subsequently ionised, for example, using electron impact ionisation, and analysed with a TOF-MS.

Here we describe a new experimental setup, recently constructed and now producing its first data, utilising this sensitive technique: a Mass-Analytical Tool for Reactions in Interstellar ICES. MATRI ${ }^{2}$ CES is an UHV system aimed at simulating conditions in dark interstellar clouds. Low temperature solid state (ice)chemistry is initiated by a microwave powered $\mathrm{H}_{2}$ discharge lamp that mimics the radiation field that is dominated by Lyman- $\alpha$ radiation and originates in space from cosmic rays excited hydrogen molecules. Such lamps have been used to study the photo-desorption, photo-dissociation, and photo-chemistry in interstellar ice analogues. ${ }^{3,37}$ Also, the initial irradiation experiments ${ }^{4-6}$ already mentioned here, were performed with this type of lamps. The performance of MATRI ${ }^{2} \mathrm{CES}$ is demonstrated by the vacuum UV photoprocessing of solid methane. $\mathrm{CH}_{4}$, a molecule that has been shown to be present in interstellar ${ }^{38-41}$ (diluted) and planetary ("pure") ices, likely forms upon hydrogenation of C-atoms, and is considered a starting point in the formation of larger hydrocarbons.

\section{SYSTEM DESCRIPTION}

MATRI $^{2}$ CES, shown schematically in Figure 1, consists of two UHV chambers: the main chamber (A) in which ices are grown and VUV irradiated, and a reflectron time-of-flight tube (B) that is used to monitor laser desorbed species mass spectrometrically. In the main chamber, ices can be deposited under fully controlled conditions at temperatures as low as $20 \mathrm{~K}$. Resistive heating allows to study ices at different temperatures, up to $300 \mathrm{~K}$. A microwave powered $\mathrm{H}_{2}$ discharge lamp is used to irradiate the ice sample with primarily Lyman$\alpha$ photons $(\lambda=121.6 \mathrm{~nm})$. This lamp (beam path, (L)) has been characterized in much detail recently by Chen et al. ${ }^{42}$

Typically, the desorption of photo-processed ice constituents is induced by using the unfocused beam of a Nd:YAG laser (Polaris II, New Wave Research, $355 \mathrm{~nm}$, 3-4 ns, beam path, (J)). Additionally, we have the opportunity to work with the fundamental or second harmonic of a Nd:YAG laser. The desorbed molecules are ionised using electron impact ionisation $(\mathrm{K})$ and detected using a reflectron time-of-flight mass spectrometer (Jordan TOF Products, Inc.).

In this section the experimental details are described, time-of-flight to mass and ice-thickness calibrations are presented, and the measurement concept is described in detail.

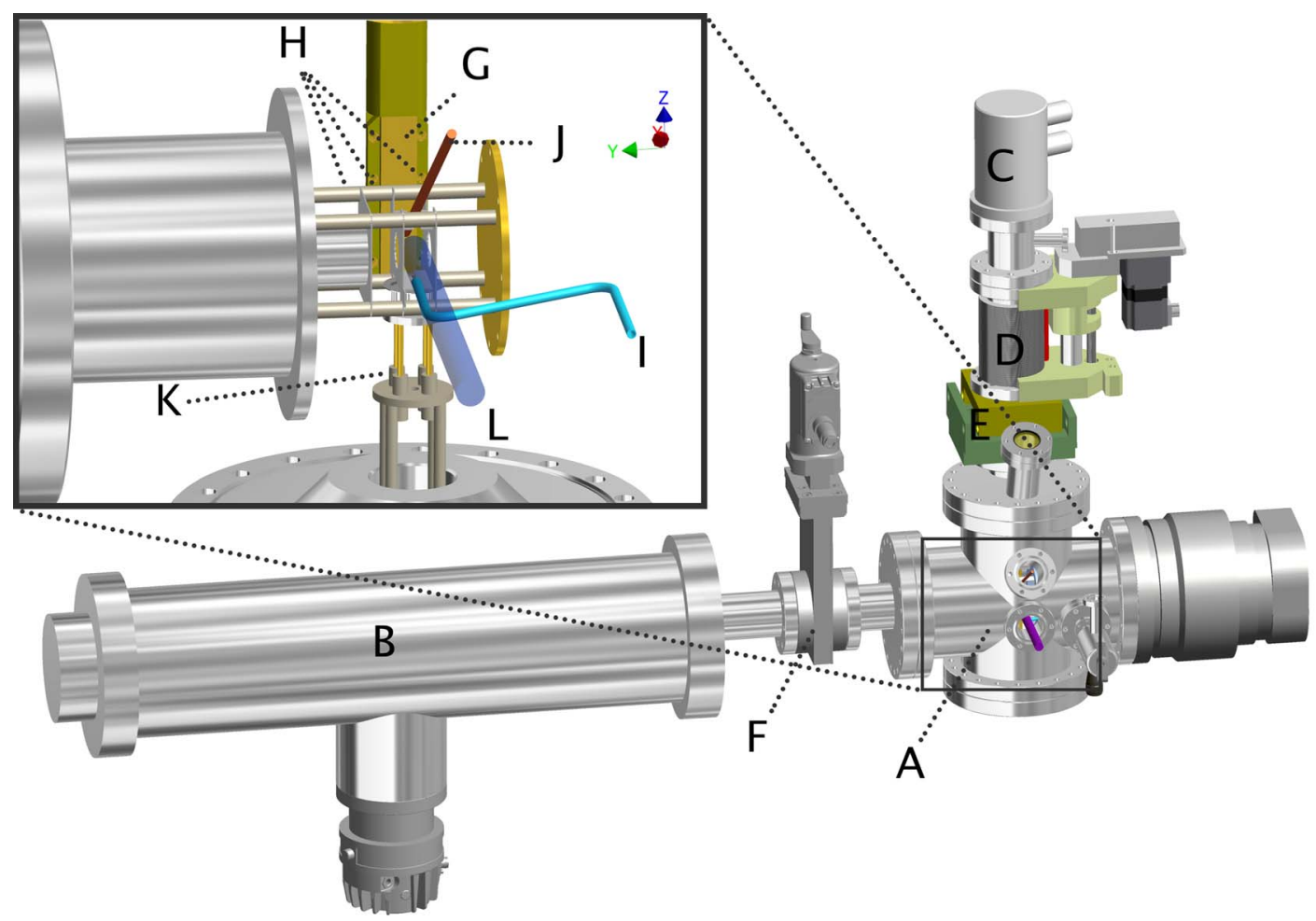

A. Main chamber

B. Flight tube

C. Cryostat

D. z-translator

E. y-translator

F. Gate valve

G. Substrate

H. Extraction optics

I. Gas inlet tube

J. Ablation laser (beam path)

K. Ionization source

(electron gun)

L. UV source (discharge lamp)

FIG. 1. Schematic drawing of MATRI ${ }^{2}$ CES (autodesk inventor), in the inset the inside of the main chamber is shown. Prevacuum pump and deposition line are not shown for clarity. 


\section{A. Main chamber}

The base pressure in the stainless steel main chamber (modified C-685 chamber, Jordan TOF Products, Inc.) is $\sim 10^{-10}$ mbar at room temperature. It is evacuated by a $550 \mathrm{l} / \mathrm{s}$ (pumping capacity for $\mathrm{N}_{2}$ ) turbo molecular pump (Varian TV551) backed up by a scroll pump (Varian TS600). On top of this chamber all ice cryogenics are mounted. An offcentred gold-coated copper cylinder $\left(250 \times 12.5^{2} \times \pi \mathrm{mm}^{3}\right)$ ending in rectangular flat sampling surface (15 mm (y-axis) $\times 75 \mathrm{~mm}$ (z-axis), $(\mathrm{G}))$ is in thermal contact with the cold finger of a helium closed-cycle cryostat (Advanced Research Systems, DE 202). Although gold is not representative for interstellar dust grains, it has several favourable properties. It is chemically inert, an effective heat conductor and effectively absorbs light in the UV range. Moreover, the ices studied here are thick and as a consequence the influence of the surface is negligible, as has been shown in studies where also other substrates were used. An indium gasket is used to obtain optimal thermal contact between the substrate and the cold finger. The substrate temperature is controlled between 20 to $300 \mathrm{~K}$ with a relative precision of $0.5 \mathrm{~K}$, using a cryogenic temperature controller (LakeShore model 331) combined with a chromel-gold/iron alloy thermocouple and a heating element. The thermocouple is inserted below the substrate and the resistive heating element is attached to the cold finger of the cryostat. A gold-coated copper thermal shield $(\sim 77 \mathrm{~K})$ surrounds $3 / 4$ of the substrate cylinder in order to reach temperatures as low as $20 \mathrm{~K}$.

The cryostat (C) is positioned on top of two bellows with two linear shift mechanisms (z- and y-direction). A motorised translation stage (UHV design, LSM64-50, McLennan, 23HT18C stepper motor with a SimStep single axis stepper system) allows to control the vertical motion (z-axis) of the substrate $( \pm 37.5 \mathrm{~mm}(\mathrm{D}))$ with variable speeds. A manual translation stage (UHV design, LDM64/64, y-axis, (E)) allows to control the horizontal motion of the substrate $( \pm 7.5 \mathrm{~mm})$. The translational stage in the vertical direction (z-axis) is necessary to improve signal-to-noise ratios by accumulating 550 mass spectra, moving the sample and realizing a "fresh" spot for the laser. The effective laser spot dimension at sample position is estimated to be $\simeq 1.25 \mathrm{~mm}$. Since the $\mathrm{H}_{2}$ discharge lamp sample coverage is equal to $2 / 5$ of its surface, the number of mass spectra is limited to 220 . The $y$-axis translation stage enables us to perform a kinetic study on a processed ice sample.

The gas phase precursor species are admitted into the main chamber with an aperture adjusted leak valve (Hositrad, (I)), through a high-vacuum stainless steel gas mixing line connected to a gas reservoir $(\sim 0.35$ liter $)$. The reservoir is then filled up to 40 mbar with $\mathrm{CH}_{4}$ (Praxair, purity 99.9995\%) — as an astronomically relevant ice constituentor with Ar (Praxair, purity 99.999\%) to further characterize the performance of MATRI ${ }^{2}$ CES. Deposition proceeds either under an angle of $\sim 85^{\circ}$ with a front deposition gas inlet tube, or following a background deposition procedure. In the latter case, a second gas inlet tube, located behind and directed away from the substrate can be used to impinge the sampling surface with gas phase molecules at random trajectories, en- suring an uniform, porous ice growth. However, as the ion optics necessarily are mounted in front of the substrate this method does not work as well as found in other studies. ${ }^{43}$ For this reason, front deposition is used, with the substrate translating up and down to obtain a quasi-uniform ice distribution following the z-axis of the sampling area.

The main chamber houses part of the orthogonal reflectron time-of-flight mass spectrometer assembly, which includes the ion extraction region (repeller plate, extraction grid, and acceleration grid, $\mathrm{H}$ ), an electron ionisation source $(\mathrm{K})$, and the ion focusing and horizontal steering plates. The sampling surface is located in the vicinity $(\sim 4 \mathrm{~mm})$ of both the ion extraction region and the electron ionisation source, in order to minimise the distance neutrals have to travel. This configuration is the most compact that can be realised in this setting and offers the advantage of a high ion transmission, increasing the detection limit.

An ultra-high vacuum magnesium fluoride $\left(\mathrm{MgF}_{2}\right)$ viewport is mounted on a DN40 flange, directly facing the sampling area, and allows to measure the ice thickness through optical laser interference $e^{44,45}$ or is used as input window of the $\mathrm{H}_{2}$ discharge lamp that is located $14 \mathrm{~cm}$ from the substrate surface. Other DN4 and DN160 flanges are used as current and gas inlets and for pressure monitoring that is realized by both a Varian IMG-300 inverted magnetron gauge (1.33 $\times 10^{-3}$ to $6.65 \times 10^{-11}$ mbar) and a Varian Agilent ConvecTorr Convection-Type Tube Sensor (atmosphere to 1.33 $\times 10^{-3}$ mbar). There is a $30^{\circ}$ diagonal DN40 conflat flange available as inlet for the Nd:YAG laser beam, which is used for the ice ablation (J). An ultra-high vacuum gate valve (DN63 conflat flange connections, VAT, (F)) separates the ion source mounting flange from the flight tube and allows to keep UHV in the flight tube during main chamber maintenance.

\section{B. Flight tube}

The stainless steel electric field-free flight tube (Jordan TOF Products, Inc.) has a base pressure better than $\sim 10^{-10}$ mbar. This pressure is monitored using a second Varian IMG300 inverted magnetron gauge. The flight tube is evacuated by a $250 \mathrm{l} / \mathrm{s}$ (pumping capacity for $\mathrm{N}_{2}$ ) turbo molecular pump (Varian TV301) backed up by a scroll pump (Varian TS600). The tube is $812.8 \mathrm{~mm}$ long and can be used in both linear and reflected mode operation. The drift tube houses the remaining part of the orthogonal reflectron time-of-flight mass spectrometer assembly. Namely, a single stage reflectron, and two Multi Channel Plates (MCP): a $40 \mathrm{~mm}$ diameter MCP reflected mode detector, and a $18 \mathrm{~mm}$ diameter MCP linear mode detector, situated behind the single stage reflectron. Both MCPs have a sub-nanosecond rise time, allowing fast ion pulse handling.

The reflectron time-of-flight mass spectrometer assembly has a D-850 angular reflectron configuration. Mass resolution of 1500 and even 4000 when used with a supersonic jet can be obtained. This is for a configuration containing the shroud, skimmer, pulsed valve, and electron gun. In the original design of MATRICES, ${ }^{20}$ the system was used in such a configuration, however due to limited transmission-from ice to ionized reaction products-we have chosen to modify the system 
to the current version. The shroud, skimmer, and pulsed valve have been removed, limiting the mass resolution (M/ $\Delta \mathrm{M}$ typically $\sim 400$ ) but increasing the overall transmission of the system.

\section{Analytical tools}

A virtual grid of $220 \times 9$ non-overlapping desorption spots is set on the ice surface prior to the analysis. For one ice sample, a maximum of 9 mass spectra can be acquired at different irradiation times, each of them averaging over 220 laser shots directed toward different fresh spots along the z-axis of the ice sample. During a mass scan, ice constituents are ablated from the sampling surface with an unfocused, frequency-tripled pulsed (355 nm, 3-4 ns) Nd:YAG laser (Polaris II, New Wave Research), running at $10 \mathrm{~Hz}$ repetition rate. The typical laser fluence used for ablation is on the order of $\sim 8-9 \mathrm{~mJ} \mathrm{~cm}^{-2}$. Four fixed mirrors (UV fused silica) and an iris (1 mm aperture) are used to steer and skim each ultraviolet laser pulse with a $30^{\circ}$ incidence angle toward a fresh spot on the z-translating ice sample. Subsequent scans can be performed once the base pressure after the previous scan reaches an asymptotic minimum value and once the sampling surface is manually shifted toward the y-axis, i.e., toward the next column of the virtual grid. There is no time delay between the desorption and the ionisation since we use a continuous $70 \mathrm{eV}$ electron ionisation source. The latter is located below the desorption plume, where an orthogonal beam of electrons intercepts the desorbing neutral gas phase species.

The main process occurring during electron impact ionisation can be written as

$$
\mathrm{M}+e^{-} \rightarrow M^{+\bullet}+2 e^{-},
$$

where $\mathbf{M}$ is the neutral species and $\mathrm{M}^{+\bullet}$ is the corresponding radical cation. Only positive ions are analysed. At around $70 \mathrm{eV}$ the kinetic energy transferred to the neutral molecules yields a large number of ions. It should be noted that $\mathrm{M}^{+} \bullet$ typically has enough internal energy to initiate fragmentation; upon electron impact ionization, more than only the molecular ion peak is expected in the time-of-flight mass spectrum. This may be helpful for structure determination but also comes with the disadvantage that different species may fragment along identical masses. The main concern of electron impact ionization therefore is that the resulting fragmentation can give ambiguous results, even when the unique fragmentation patterns for a large set of astrophysically relevant molecules is available from the NIST Standard Reference Database. ${ }^{46}$ This is a well-known restriction when applying electron impact ionisation.

Ions are generated between the repeller plate and the extraction grid, which are perpendicular to the sampling surface plane. During the ionisation process, these two elements carry the same voltage $(1050 \mathrm{~V})$, after which the extraction grid potential is lowered by $100 \mathrm{~V}(4 \mu \mathrm{s})$, thus orthogonally extracting the generated ions to the electric field-free flight tube. The latter allows ions to drift and to separate based on their mass/charge $(\mathrm{m} / \mathrm{z})$ ratios. At the end of the flight tube, the ions are back-reflected for improved mass resolution before

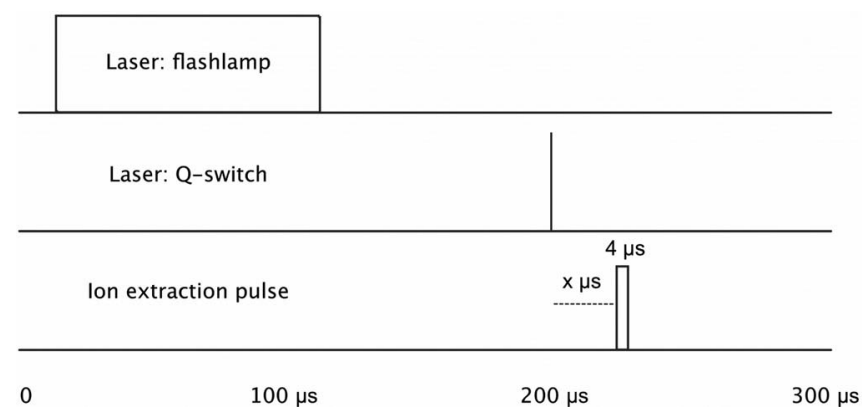

FIG. 2. Typical time sequence for a diagnostic run.

detection using the $40 \mathrm{~mm}$ MCP detector. The reflectron actually compensates the initial kinetic energy distribution by sharpening ion packets of same $\mathrm{m} / \mathrm{z}$ ratio. The ion signal is then recorded by a PCIe digitizer card ( 8 bit, 3 GS/s, Gage CobraMax CS13G8).

The timing sequence of the laser pulse, the ion extraction pulse, and the data acquisition trigger is controlled by a delay/pulse generator (DG535, GPIB interface, Stanford Research System). Instrument control, data acquisition, and data processing are performed using LabVIEW 8.6 (National Instruments). A typical timing sequence for a separate analysis run is shown in Figure 2; the flash lamp and Q-switch of the laser are triggered, and after a variable time (typically on the order of $\mu \mathrm{s}$ ) the extraction pulse extracts ions into the timeof-flight tube. Depending on the exact difference between the laser pulse and the ion extraction pulse, spatially different parts of the desorption plume can be probed.

\section{Calibration experiments}

The time-of-flight mass (Sec. II D 1) and ice thickness (Sec. II D 2) calibration follow standard procedures that are mentioned here for completeness.

\section{Mass calibration}

A 3-point internal calibration is performed for each timeof-flight spectrum using the fragment ion peaks and/or the molecular ion peaks corresponding to the initial ice sample constituents, and by solving the following equation: ${ }^{47}$

$$
\mathrm{TOF}=A+B \times \sqrt{\mathrm{m} / \mathrm{z}}+C \times \mathrm{m} / \mathrm{z},
$$

with $A, B$, and $C$ the three parameters to find. The first two terms correspond to the conventional time-of-flight equation and the last term is introduced to improve the mass accuracy over the whole $\mathrm{m} / \mathrm{z}$ range. The mass coverage used in our experiments extends from 0 to $300 \mathrm{~m} / \mathrm{z}$, here the focus is on masses up to $100 \mathrm{~m} / \mathrm{z}$ and a typical mass resolution with $\mathrm{M} / \Delta \mathrm{M} \sim 320$ to $\sim 400$.

\section{Thickness calibration}

During deposition, the ice thickness can be monitored by optical laser interference ${ }^{44,45}$ using an intensity stabilised helium neon (He-Ne) laser (Thorlabs HRS015). The laser beam strikes the sampling surface at an incident angle $\theta \simeq 88.5^{\circ}, \theta_{1}$ is defined as the angle of reflection within the ice sample. The 


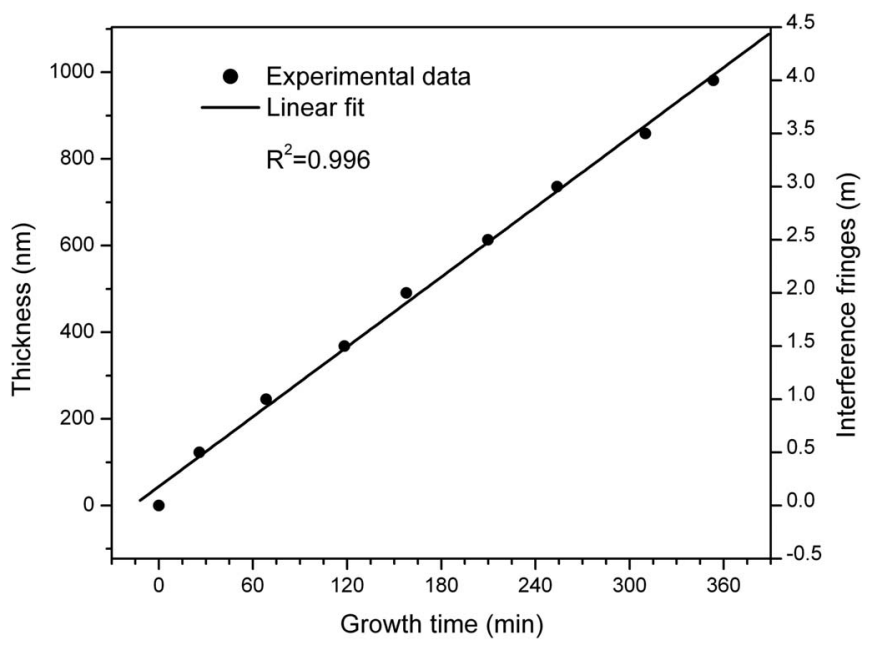

FIG. 3. Linear fit to subsequent interference fringes - minima and maxima (m) - and the corresponding thickness vs. deposition time.

reflected light is thereafter collected and converted to a digital signal by a photodiode (Thorlabs PDA36A). The ice thickness $d$ can be expressed as a function of interference fringes (m):

$$
d=\frac{\mathrm{m} \lambda}{2 \mathrm{n}_{1} / \mathrm{n}_{0} \cos \theta_{1}},
$$

where $\lambda$ corresponds to the wavelength of the He-Ne laser $(632.8 \mathrm{~nm}), \mathrm{n}_{0}$ and $\mathrm{n}_{1}$ are the refractive indices of vacuum and growing ice, and $\theta_{1}$ is the angle of refraction. A quantitative characterisation of the ice thickness during deposition is possible by correlating the interference fringe pattern of subsequent minima and maxima $(\mathrm{m})$ and the corresponding deposition time. A linear fit to this data provides the best representation of the deposition rate as depicted in Figure 3.

In this example of $\arg$ on $(n=1.29 \text { at } 20 \mathrm{~K})^{48}$ the growth rate is $2.68 \pm 0.07 \mathrm{~nm} / \mathrm{min}$. Icy layers of dust grains in the ISCM typical range up to $\sim 100 \mathrm{ML}$, assuming that a monolayer has a thickness of $3 \AA$, relevant ice-thicknesses would range up to $30 \mathrm{~nm}$. The obtained growth rates can then be adapted to astronomically relevant ice coverages.

A similar growth rate has been realized for methane during deposition. In the methane experiments the z-translator cycles up and down to ensure homogenous ice growth along that coordinate. A slight tilt of the sample, combined with surface roughness, did not allow to perform a laser interference calibration. Therefore, we have estimated the z-direction coverage of the deposition tube. The previously determined deposition rate for argon ice has been used for ice growth without cycling the z-translator. Combining this estimate with the known deposition rate, we have estimated the ice thickness of our typically deposited ice to be $15 \pm 1 \mathrm{~nm}$.

\section{E. Experimental concept}

Methane is let into the mixing line up to a typical pressure of 40 mbar. In order to ensure homogeneous ice growth in the z-direction, the translator cycles up and down during this stage. The y-translator is fixed in the middle. Subse- quently, the needle valve is opened up to the calibrated setting aperture, slowly leaking $\mathrm{CH}_{4}$ into the chamber. Methane molecules will strike the cold substrate, stick, growing a multilayer ice on top. At a sample temperature of $20 \mathrm{~K}$, pressure read during deposition is typically $2 \times 10^{-8}$ mbar. The deposition is stopped after about $15 \mathrm{~min}$, and the typical ice thickness reached is $\sim 15 \mathrm{~nm}(\sim 50 \mathrm{ML})$. After deposition, the sample is moved using the y-translator toward the first column of the virtual grid, as described in Sec. II C. A total of 220 mass spectra are averaged at a $10 \mathrm{~Hz}$ repetition rate, without UV irradiation, to record a mass spectrum for the unprocessed ice. The constant vertical speed of the substrate allows the fixed laser beam to hit different "fresh" spots on the ice sample. The first averaged mass spectrum corresponds to the reference, i.e., pure methane mass spectrum and serves at validating the different settings of MATRI ${ }^{2} \mathrm{CES}$ (e.g., alignments, timing and applied potentials). Every single laser shot induces desorption of the ice constituents that are ionised and detected mass spectrometrically. After obtaining the mass spectra, the sample is re-positioned in the starting position.

VUV ice photolysis is started by igniting the $\mathrm{H}_{2}$ discharge lamp. A wide range of emission spectra and fluxes is accessible by mixing the residual gases with quenching species such as noble gases, by adjusting the partial pressure of molecular hydrogen inside the lamp, and by adjusting the microwave power. In order to further control the emission spectrum and fluxes during ice processing, settings as

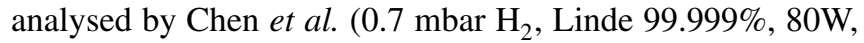
F-type quartz-lamp geometries) are used. ${ }^{42}$ The VUV emission spectrum of the lamp is in the $115-170 \mathrm{~nm}$ range, and peaks at $121.6 \mathrm{~nm}$. Moreover, there is a clear emission band around $160 \mathrm{~nm}$. For the moment we will not focus on the exact lamp settings too much, as the goal of this work is to present a new analysis method for the resulting solid state photoproducts. The photon flux in this range is generally estimated to be $\sim 5 \times 10^{13}$ photons $\mathrm{s}^{-1} \mathrm{~cm}^{-2}$, which corresponds to a fluence in dense molecular clouds over a period of $10 \times 10^{6}$ of years. ${ }^{49}$ Solid methane absorbs VUV in the range below $\sim 130 \mathrm{~nm} .{ }^{50}$ Since these photons have a significantly higher energy than the bond dissociation energy of methane, radicals will be formed in the ice. Radical/radical recombination and radical/neutral reactions will result in more complex species such as ethane $\left(\mathrm{C}_{2} \mathrm{H}_{6}\right) .{ }^{51-53}$ At different predefined irradiation times, $0.5,1,2,4,8,16,32$, and $64 \mathrm{~min}$, the VUV emission is stopped and a time-of-flight spectrum is recorded. In this way, the formation of complex species during VUV irradiation can be investigated.

This experimental concept has clear advantages with respect to the early experiments, in which the residue had to be studied, ex situ, in air, and at room temperature. MATRI ${ }^{2} \mathrm{CES}$ allows to record mass spectra at astronomically relevant temperatures and under fully controlled conditions in situ. Thermal processing can be excluded, and data are recorded basically in real time. In order to characterize the experimental performance of MATRI ${ }^{2} \mathrm{CES}$, this paper focuses on a bottomup scenario, starting from individual precursors, rather than a mixture. The ultimate goal of this setup, will be to characterize mass spectra of ices as complex as used in the initial radiation experiments. 


\section{RESULTS}

In this section we present the behaviour of methane ice under influence of VUV radiation and the validation of the diagnostic concept. This section is divided into three subsections; Subsection III A focusses on interpreting the obtained spectra after VUV irradiation qualitatively, Subsection III B describes the kinetics following a fitting procedure of the small species, and in Subsection III C the experimental constraints are discussed.

\section{A. Kinetic study: Qualitative approach}

The photo-chemical evolution, i.e., fluence dependence of the mass spectra during the $64 \mathrm{~min}$ of irradiation is presented in Figure 4. Clearly visible in the reference mass spectrum $(\mathrm{t}=0 \mathrm{~min})$, is the fragmentation pattern of $\mathrm{CH}_{4}$ resulting from the electron impact. In addition to this fragmentation pattern, low intensity peaks appear at $\mathrm{m} / \mathrm{z} 26\left(\mathrm{C}_{2} \mathrm{H}_{2}^{+}\right), 27$ $\left(\mathrm{C}_{2} \mathrm{H}_{3}^{+}\right), 28\left(\mathrm{C}_{2} \mathrm{H}_{4}^{+}\right)$and at 32, 33, 48, and 49. The last four peaks can be attributed to $\mathrm{CH}_{4}^{+}$-clusters, with $\mathrm{m} / \mathrm{z} 33$ and 49 with a combination of ${ }^{13} \mathrm{CH}_{4}^{+}$containing clusters and (protonated) clusters. These clusters are the result of the pure nature of the methane ice, and after only $8 \mathrm{~min}$ of irradiation the clusters disappear in the spectra. A substantial amount of larger species has been formed.

Directly after the onset of the irradiation, more complex species such as ethane are formed, as can be deduced from the typical mass fragmentation pattern. This behaviour is in agreement with previous observations using IR spectroscopy of energetically processed methane ice..$^{51-53}$

A comparison of the mass spectra shows that the low intensity peaks observed in the reference mass spectrum $(\mathrm{t}=0$ min) are significantly less pronounced than the peaks arising upon VUV irradiation. From the increasing signal at higher masses, it is clear that for higher fluences the complexity of hydrocarbon species formed in the ice further increases.

A detailed look at the final acquired mass spectrum is presented in Figure 5. In particular at higher masses $(\mathrm{m} / \mathrm{z}>48)$, exact quantification of the individual detected peaks is chal-

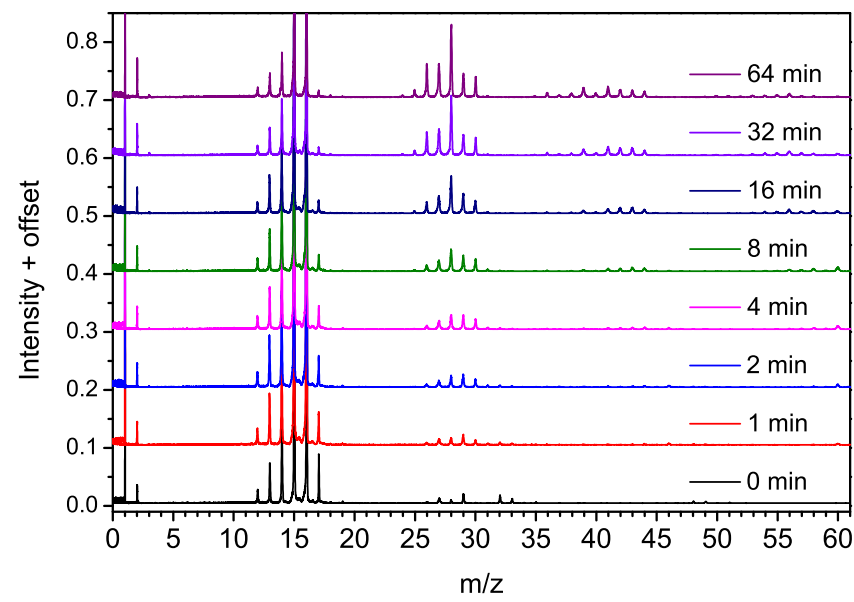

FIG. 4. Mass spectra of $\sim 15 \mathrm{~nm}$-thick methane ice $\left(\mathrm{CH}_{4}\right)$, deposited at $20 \mathrm{~K}$ (220 averages measuring time $\simeq 22$ s per spectrum) at different VUV irradiation times, using a microwave $\mathrm{H}_{2}$ discharge lamp. The total fluence ranges from $3 \times 10^{15}$ photons $\mathrm{cm}^{-2}(1 \mathrm{~min})$ to $1.9 \times 10^{17}$ photons $\mathrm{cm}^{-2}(64 \mathrm{~min})$, corresponding roughly with $10^{7}$ years in a dark interstellar cloud.

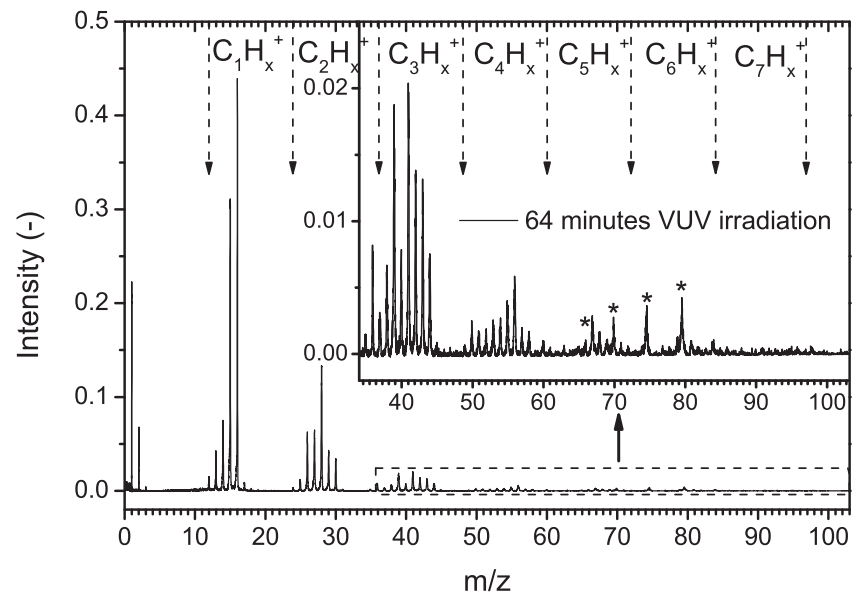

FIG. 5. Mass spectrum of $\sim 15 \mathrm{~nm}$-thick methane ice $\left(\mathrm{CH}_{4}\right)$, deposited at $20 \mathrm{~K}$ (220 averages, measuring time $\simeq 22 \mathrm{~s}$ ). This ice is processed for 64 min using the $\mathrm{H}_{2}$-discharge lamp. The inset shows a detailed version of the high mass part of the spectrum. Peaks with an asterisk cannot be related to a specific species and are likely due to the fact that the present experiment lacks a skimmer between the photoprocessing and detection units.

lenging, as similar mass peaks may be due to different species, but even then it is clear that peaks show up in the $\mathrm{C}_{6} \mathrm{H}_{x}^{+}$mass range, evidencing the formation of larger species. Following Eq. (1), these peaks can only originate from species either with that size or larger.

From this figure it becomes clear that with increasing fluence also the level of molecular complexity in the ice increases. Clearly, a reaction chain is followed in which newly formed species can recombine with radicals, forming even more complex species. For a relatively short irradiation time - in the initial experiments ices were irradiated for days ${ }^{4-6}$ - already rather large species with up to seven carbon atoms can be found. This is very comparable to findings concluded from VUV irradiation of pure methanol ice, ${ }^{3}$ yielding new species with up to 10 atoms. There further analysis was hindered by spectral confusion and mass spectrometric limitations (mass range). Specifically, the latter point is not an issue with the TOF system used here. However, as stated before, identical fragmentation products (or masses) for different species make an analysis complicated, but not impossible.

\section{B. Kinetic study: Quantitative approach}

Solid methane has previously been irradiated using a $\mathrm{H}_{2}$ microwave discharge lamp, using IR spectroscopy as analytical technique, and also here larger hydrocarbons have been detected. ${ }^{51-53}$ In order to quantify the kinetics upon solid $\mathrm{CH}_{4}$ irradiation (Figure 4) we fitted the mass spectra recorded at different irradiation times.

The overall time-of-flight spectra are first masscalibrated as described in Sec. II D 1. Subsequently, the mass spectra are baseline corrected, by fitting a baseline through an array of points where no signal is present. The individual peaks are then integrated to obtain the total intensity of every single mass. This intensity is proportional to the number of ions detected.

Each mass spectrum obtained at different irradiation times is regarded as a linear combination of $n$ individual 

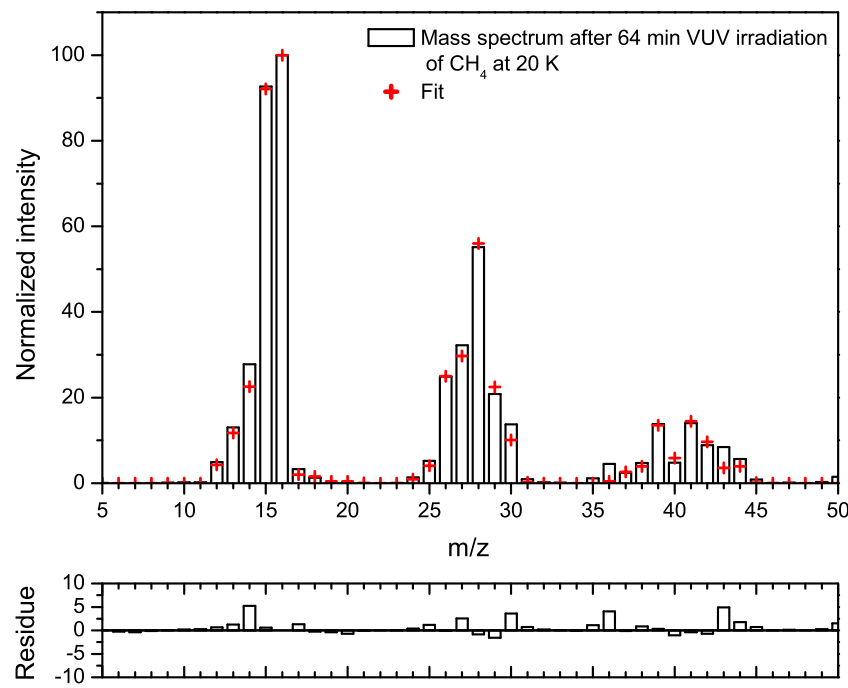

FIG. 6. Fit of the mass spectrum obtained after 64 min VUV photolysis of $\mathrm{CH}_{4}$ at $20 \mathrm{~K}$ in linear combination of $70 \mathrm{eV}$ electron impact mass spectrum (NIST Standard Reference Database 69: NIST Chemistry WebBook) ${ }^{46}$ and electron impact cross sections (NIST: Electron-Impact Cross Section Database). ${ }^{55}$

compounds present in the ablated material:

$$
\mathrm{MS}_{t}=\sum_{i=1}^{n} a_{i} \times \sigma_{i} \times \mathrm{MS}_{i}
$$

where $\mathrm{MS}_{t}$ is the mass spectrum acquired at a specific irradiation time $t, a_{i}$ corresponds both to the fitting parameter and to the mole fraction of a constituent $i, \sigma_{i}$, and $\mathrm{MS}_{i}$ are the corresponding electron impact cross section and the reference electron impact mass spectrum, respectively. The fitting procedure is driven by MATLAB 7.9.0 (R2009b), and uses the Nelder-Mead optimisation algorithm. ${ }^{54}$ Figure 6 shows an example of the fitting procedure of the mass spectrum discussed before after $64 \mathrm{~min}$ VUV photolysis of $\mathrm{CH}_{4}$ at $20 \mathrm{~K}$. The red crosses in the top panel of the figure show the best fit of Eq. (4) to the mass spectrum together with the residuals in the bottom panel. The fitting library is shown in Table I, and consists of all hydrocarbons containing less than four carbon atoms as well as possible contaminants.

TABLE I. Electron-impact cross sections of species considered in the mass fitting routine.

\begin{tabular}{lcc}
\hline \hline Name & Formula & $\sigma_{70 e V}\left(\AA^{2}\right)^{55}$ \\
\hline Methane & $\mathrm{CH}_{4}$ & 3.524 \\
Ethane & $\mathrm{C}_{2} \mathrm{H}_{6}$ & 6.422 \\
Ethylene & $\mathrm{C}_{2} \mathrm{H}_{4}$ & 5.115 \\
Acetylene & $\mathrm{C}_{2} \mathrm{H}_{2}$ & 4.374 \\
Propane & $\mathrm{C}_{3} \mathrm{H}_{8}$ & 8.619 \\
Propene & $\mathrm{C}_{3} \mathrm{H}_{6}$ & 8.736 \\
Allene & $\mathrm{C}_{3} \mathrm{H}_{4}$ & 8.080 \\
Propyne & $\mathrm{C}_{3} \mathrm{H}_{4}$ & 7.662 \\
Nitrogen & $\mathrm{N}_{2}$ & 2.508 \\
Oxygen & $\mathrm{O}_{2}$ & 2.441 \\
Water & $\mathrm{H}_{2} \mathrm{O}$ & 2.275 \\
\hline \hline
\end{tabular}

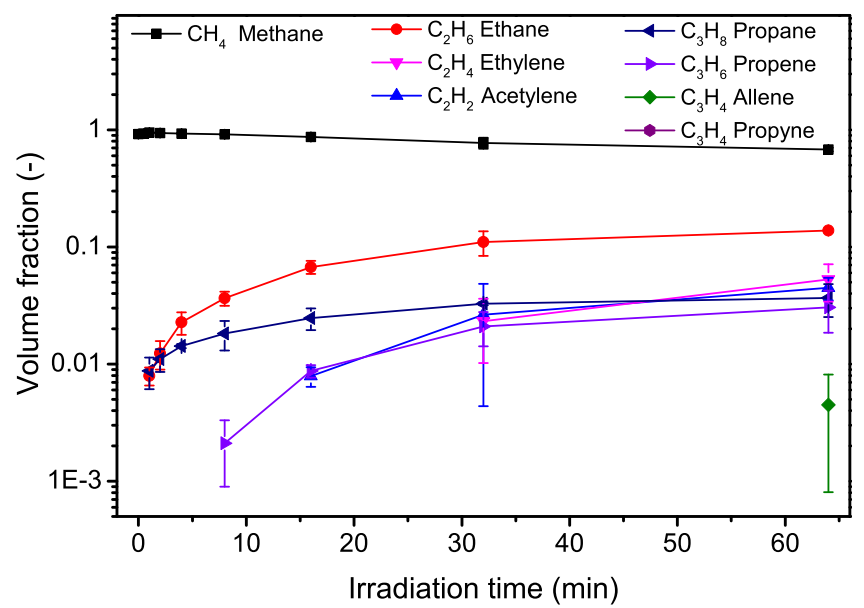

FIG. 7. Evolution of small hydrocarbons during the first $64 \mathrm{~min}$ of VUV irradiation. The error bar is based on three times the standard deviation, the large error at $\mathrm{t}=32 \mathrm{~min}$ is due to a smaller statistical sample. Propyne is included in the fitting library, but is not observed.

Larger species are excluded in the fitting library, since the accuracy of the fit above $\mathrm{m} / \mathrm{z} 48$ is similar to the intensity of the individual peaks.

The photo-chemical evolution of the species, fitted from the kinetics mass spectra (Figure 5) is shown in Figure 7. The same fitting library, Table I, is used for all mass spectra at different irradiation times. The degeneracy of the fit has been examined by using different initial guesses for all parameters $\left(a_{i}\right)$; the same minimum was found, resulting in the same set of abundances. Photodesorption has been neglected and the assumption has been made that the sum of all species is normalised. Propyne is not observed in the 64 min irradiation, but most likely can be formed if the propene abundance further increases during longer irradiation time scales. We are confident about the fit, since the formation trend of the species makes sense from a chemical perspective.

In previous photo-processing studies of solid methane, the formation of ethane and propane were observed, and clearly larger aliphatic chains were also formed. ${ }^{51-53}$ As the vibrational modes of different hydrocarbons are rather similar, their absorption profiles overlap substantially. MATRI ${ }^{2} \mathrm{CES}$ has the capability of providing additional insights in the formation networks of such species, and this will be discussed in a forthcoming paper.

\section{Validation experiments}

In this section, the performance of the system is validated, as MATRI ${ }^{2}$ CES is crossing untouched areas. Subsections III C 1-III C 3, the influence of the laser ablation wavelength and the extraction time will be discussed, as well as the detection limit and the typical mass resolution obtained.

\section{Ablation wavelength dependance}

In order to investigate the influence of laser wavelength, we have repeated experiments for three clearly different wavelengths. The fundamental of a Nd:YAG laser, frequency doubled and tripled: 1064, 532, and $355 \mathrm{~nm}$, respectively, have all been used to ablate pure $\mathrm{CH}_{4}$ ice. At these three 


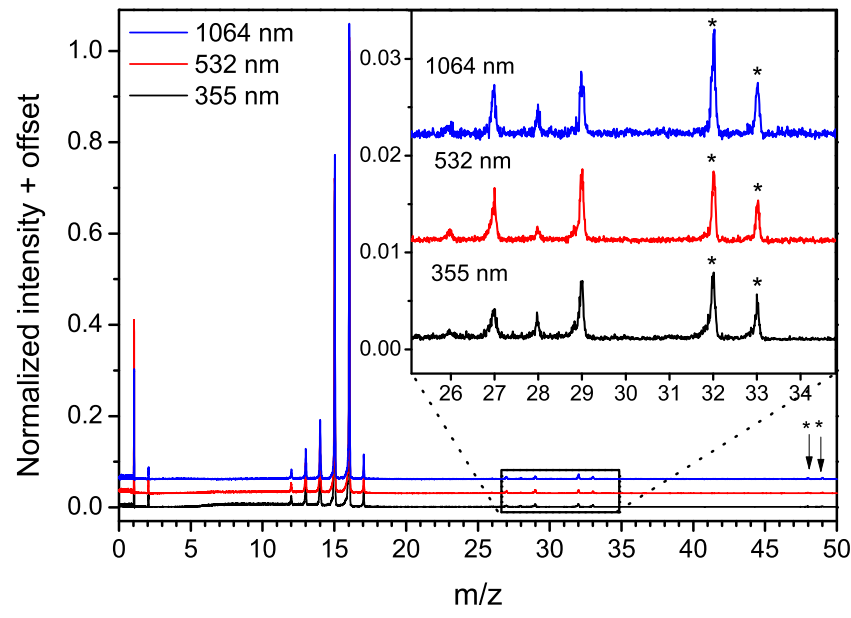

FIG. 8. Mass spectra of ablated $\mathrm{CH}_{4}$ ice using different ablation wavelengths. Peaks marked with an asterisk (*) are attributed to $\mathrm{CH}_{4}$ clusters. The inset shows a zoom-in of the part containing additional peaks. The shown mass spectra are normalised to the $\mathrm{m} / \mathrm{z} 16$ peak for comparison.

wavelengths, $\mathrm{CH}_{4}$ has no clear overlap with electronic transitions. Common interstellar ice constituents such as $\mathrm{H}_{2} \mathrm{O}, \mathrm{CH}_{3} \mathrm{OH}, \mathrm{CO}$, e.g., do not exhibit transitions at these wavelengths either. The resulting mass spectra are shown in Figure 8.

Clearly visible in these spectra, is the fragmentation pattern of $\mathrm{CH}_{4}$ resulting from the electron gun ionisation. In addition to the fragmentation pattern, low intensity peaks appear at $\mathrm{m} / \mathrm{z} 26\left(\mathrm{C}_{2} \mathrm{H}_{2}^{+}\right), 27\left(\mathrm{C}_{2} \mathrm{H}_{3}^{+}\right), 28\left(\mathrm{C}_{2} \mathrm{H}_{2}^{+}\right)$and $\mathrm{m} / \mathrm{z} 32$, 33,48 , and 49 originate from $\mathrm{CH}_{4}$ clusters, as previously discussed, a combination of ${ }^{13} \mathrm{CH}_{4}^{+}$containing clusters and protonated clusters. The intensity of the observed peaks at $\mathrm{m} / \mathrm{z}$ $26,27,28$, and 29 slightly differs between different wavelengths, however the same peaks are observed. The amount of energy per pulse to induce desorption of the ice differs strongly with wavelength, as is expected from the properties of gold. Gold absorbs UV photons with a high efficiency, while only a small fraction of IR photons is absorbed. This experiment shows that the ablation process in the examined wavelength range is similar. We have opted to use $355 \mathrm{~nm}$ for the VUV irradiation experiments, since applying different wavelengths does not affect the final mass spectrum.

\section{Ion extraction time}

As mentioned before, the time sequence is controlled by the DG535 pulse generator. After the laser pulse induces desorption, a plume of desorbing ice constituents is directed toward both the ion optics and ionisation source. Different time delays between the laser pulse and the ion extraction pulse are taken to probe the different parts of the desorption plume and ultimately to optimise the extraction timing and the signalto-noise ratio (Figure 2, $\mathrm{x} \mu \mathrm{s}$ ). Figure 9 shows a typical ion extraction time scan from an argon ice with a thickness of $\sim 60 \mathrm{ML}$. Argon is used here, instead of $\mathrm{CH}_{4}$, since its isotopes offer a direct way to estimate the sensitivity level of MATRI $^{2}$ CES. Each of these spectra is the average of 220 individual spectra.

In Figure 10, the integrated area of the ${ }^{40} \mathrm{Ar}^{+}$is plotted versus the ion extraction time. Depending on laser flu-

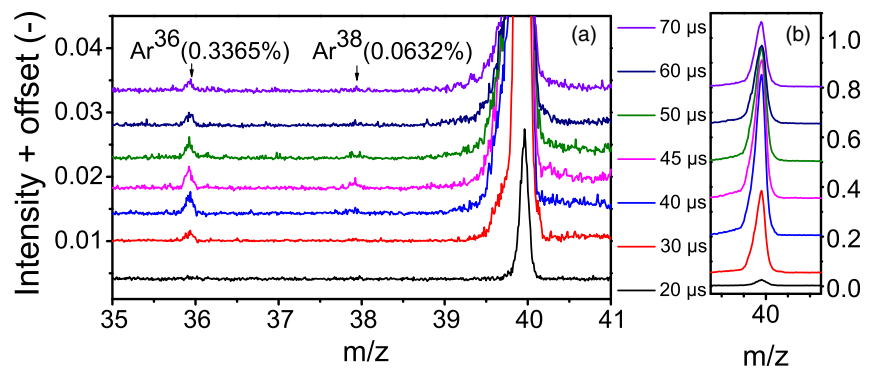

FIG. 9. Mass spectra of ablated argon ice with a thickness of 60 ML. The time label indicates time difference between laser pulse and ion extraction of ions to the flight tube. Panel (a) shows the overview, focussing on the argon isotopes, ${ }^{36} \mathrm{Ar}^{+}$and ${ }^{38} \mathrm{Ar}^{+}$. Panel (b) shows the main ${ }^{40} \mathrm{Ar}^{+}$peak.

ence, and ice thickness the optimal extraction time can differ slightly, with typical values in the $15-70 \mu$ s range. In this example, the optimal time difference between the laser pulse and the ion extraction pulse is $45 \mu \mathrm{s}$.

\section{Detection limit and mass resolution}

The detection limit of the instrument is estimated by ablating different thin films of pure argon ice at $20 \mathrm{~K}$. In contrary to the deposition of methane, the z-translator does not cycle up and down during deposition, providing a more accurate estimation of the ice-thickness. Argon has the additional advantage that it does not fragment upon electron impact ionization, and the two existing stable isotopes aid in determining the detection limit of MATRI ${ }^{2} \mathrm{CES}$. The experimental conditions are the same as with the photolysis experiments. By monitoring the two stable isotopic peaks, ${ }^{38} \mathrm{Ar}^{+}$and ${ }^{36} \mathrm{Ar}^{+}$, and combining this observation with their natural abundances, $0.063 \%$ and $0.337 \%$, an estimate of the detection limit can be obtained.

The detection limit has a strong dependence on the settings of the DAQ-card. In this case we try to derive the detection limit of MATRI ${ }^{2} \mathrm{CES}$ under the same conditions as with the VUV irradiation experiments. The laser ablation intensity is kept constant over the different experiments, only the ion extraction time is optimised for every different ice thickness.

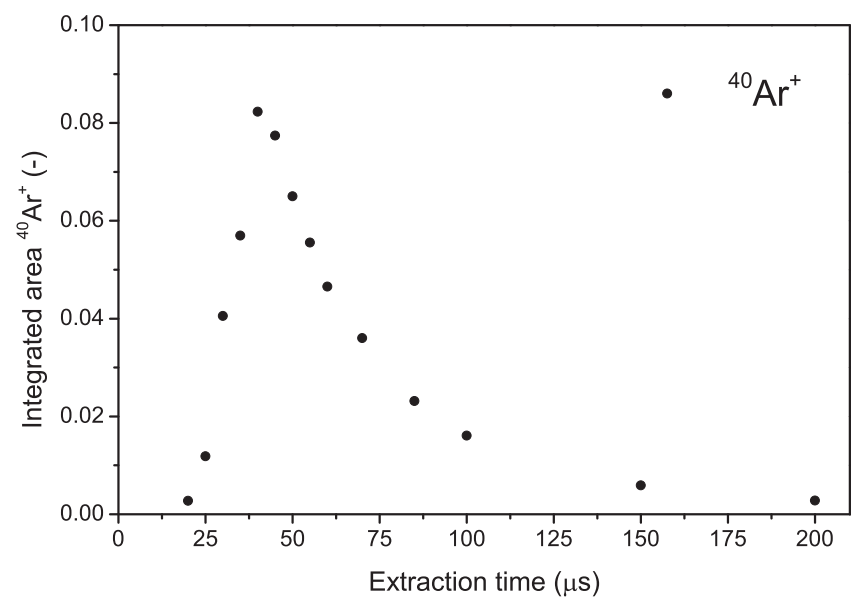

FIG. 10. Integrated area of the ${ }^{40} \mathrm{Ar}^{+}$peak versus extraction time, the time difference between the laser pulse and the ion extraction pulse. 


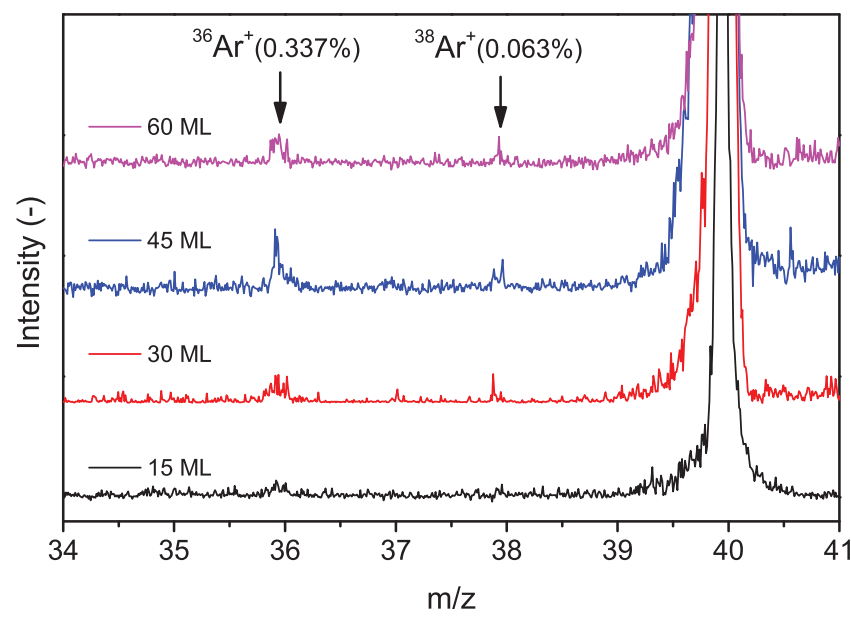

FIG. 11. Mass spectra of laser ablated argon ice of different layer thickness. The two stable argon isotopes are used to estimate the detection limit of MATRI $^{2}$ CES.

In Figure 11 the results for different argon ice thicknesses are shown.

For an ice thickness of $30 \mathrm{ML}$, the ${ }^{38} \mathrm{Ar}^{+}$becomes clearly visible. This inclines that the typical detection limit using these specific settings, is equal to or better than $30 \mathrm{ML}$ $\times 0.063 \% \leq 0.02 \mathrm{ML}$. This value is significantly better than other solid state techniques such as IR spectroscopy.

Analysing ${ }^{40} \mathrm{Ar}^{+}$by taking the FWHM, the mass resolution obtained for ablated argon ice is $M / \Delta M \sim 400$. For solid $\mathrm{CH}_{4}$ the mass resolution for the molecular ion peak is $\mathrm{M} / \Delta \mathrm{M}$ $\sim 320$. This mass resolution is more than sufficient to apply for heavier species.

\section{CONCLUSIONS}

MATRI $^{2}$ CES, a new ultra-high vacuum experimental setup, introduced here, combines laser desorption with TOF mass spectrometry to study photo induced chemical processes in interstellar ice analogues, taking advantage of the high sensitivity of mass spectrometric techniques. Utilising this new in situ analysis method, we have demonstrated that VUV photo-processing $\left(\sim 1.9 \times 10^{17}\right.$ photons $\left.\mathrm{cm}^{-2}\right)$ of solid $\mathrm{CH}_{4}$ ice yields species with at least 6 carbon atoms. With a more quantitative approach, using electron induced fragmentation patterns listed in the NIST database, we demonstrate that we can trace the formation routes of small carbon species in our methane ice upon VUV photo-processing. Electron impact cross sections and fragmentation patterns for different molecules are known and can be used to interpret the obtained mass spectra. The advantage of electron impact ionisation over, for example, REMPI is its general applicability. REMPI strongly depends on photon cross sections, while the cross sections for electron impact ionisation are in the same order of magnitude. Additional information on individual reactions in the photochemistry can be obtained using, for example, deuterated species and ${ }^{13} \mathrm{C}$-labelling.

The results presented here show the potential of this new experimental setup for astrochemistry. It provides an additional, complementary analysis method, next to the regular solid state techniques, of examining the processes occurring in the solid phase. We expect that MATRI ${ }^{2} \mathrm{CES}$ will be able to bridge solid state reaction in interstellar ices as studied so far, i.e., for relatively small molecules, to larger species of (pre)biotic interest. MATRI ${ }^{2} \mathrm{CES}$ also holds the potential to provide rate constants and branching ratios of photochemical reactions.

The experiments performed here on $\mathrm{CH}_{4}$ ice are of limited astronomical interest. Methane ice exists, ${ }^{38-41}$ but in the ISCM it is not found in a pure form. For this reason, also $\mathrm{CH}_{4}$ in $\mathrm{H}_{2} \mathrm{O}$ will be studied. The ultimate aim of MATRI ${ }^{2} \mathrm{CES}$, is to investigate in situ the formation of complex organic molecules, for example, sugars and amino-acids, under the photo-processing of interstellar ice analogous. Can these species be formed in photo-processed interstellar ice analogous? In which environments (ice composition, temperature, VUV fluence) will they form most efficient? With the settings described here such objectives now come within reach, and the goal for the nearby future is to reproduce previous mixed bulk ice experiments, but now for fully controlled experimental conditions.

\section{ACKNOWLEDGMENTS}

This work was supported by NOVA, the Netherlands Research School for Astronomy, a Vici grant from the Netherlands Organisation for Scientific Research (NWO), and the European Community 7th Framework Programme (FP7/2007-2013) under Grant Agreement No. 238258. Support for J.-B. Bossa from the Marie Curie Intra-European Fellowship (FP7-PEOPLE-2011-IEF-299258) is gratefully acknowledged. We thank M.J.A. Witlox for his significant part in construction of the instrument.

${ }^{1}$ E. Herbst and E. F. van Dishoeck, Annu. Rev. Astron. Astrophys. 47, 427 (2009).

${ }^{2}$ R. T. Garrod and E. Herbst, Astron. Astrophys. 457, 927 (2006).

${ }^{3}$ K. I. Öberg, R. T. Garrod, E. F. van Dishoeck, and H. Linnartz, Astron. Astrophys. 504, 891 (2009).

${ }^{4}$ G. M. Muñoz Caro, U. J. Meierhenrich, W. A. Schutte, B. Barbier, A. Arcones Segovia, H. Rosenbauer, W. H.-P. Thiemann, A. Brack, and J. M. Greenberg, Nature 416, 403 (2002).

${ }^{5}$ M. P. Bernstein, J. P. Dworkin, S. A. Sandford, G. W. Cooper, and L. J. Allamandola, Nature 416, 401 (2002).

${ }^{6}$ R. Briggs, G. Ertem, J. P. Ferris, J. M. Greenberg, P. J. McCain, C. X. Mendoza-Gomez, and W. A. Schutte, Origins Life Evol. B. 22, 287 (1992).

${ }^{7}$ M. Nuevo, U. J. Meierhenrich, G. M. Muñoz Caro, E. Dartois, L. d'Hendecourt, D. Deboffle, G. Auger, D. Blanot, J.-H. Bredehöft, and L. Nahon, Astron. Astrophys. 457, 741 (2006).

${ }^{8}$ M. Nuevo, U. J. Meierhenrich, L. d'Hendecourt, G. M. Muñoz Caro, E. Dartois, D. Deboffle, W. H.-P. Thiemann, J.-H. Bredehöft, and L. Nahon, Adv. Space Res. 39, 400 (2007).

${ }^{9}$ J. E. Elsila, J. P. Dworkin, M. P. Bernstein, M. P. Martin, and S. A. Sandford, Astrophys. J. 660, 911 (2007).

${ }^{10}$ M. Nuevo, G. Auger, D. Blanot, and L. d'Hendecourt, Origins Life Evol. B. 38, 37 (2008).

${ }^{11}$ Y.-J. Chen, M. Nuevo, T.-S. Yih, W.-H. Ip, H.-S. Fung, C.-Y. Cheng, H.-R. Tsai, and C.-Y. R. Wu, Mon. Not. R. Astron. Soc. 384, 605 (2008).

${ }^{12}$ P. de Marcellus, C. Meinert, M. Nuevo, J.-J. Filippi, G. Danger, D. Deboffle, L. Nahon, L. d'Hendecourt, and U. J. Meierhenrich, Astrophys. J. Lett. 727, L27 (2011).

${ }^{13}$ K. Kobayashi, T. Kasamatsu, T. Kaneko, J. Koike, T. Oshima, T. Saito, T. Yamamoto, and H. Yanagawa, Adv. Space Res. 16, 21 (1995).

${ }^{14}$ R. L. Hudson, M. H. Moore, J. P. Dworkin, M. P. Martin, and Z. D. Pozun, Astrobiology 8, 771 (2008). 
${ }^{15}$ A. Lafosse, M. Bertin, A. Domaracka, D. Pliszka, E. Illenberger, and R. Azria, Phys. Chem. Chem. Phys. 8, 5564 (2006).

${ }^{16}$ P. D. Holtom, C. J. Bennett, Y. Osamura, N. J. Mason, and R. I. Kaiser, Astrophys. J. 626, 940 (2005)

${ }^{17}$ J.-B. Bossa, F. Duvernay, P. Theulé, F. Borget, L. d'Hendecourt, and T. Chiavassa, Astron. Astrophys. 506, 601 (2009).

${ }^{18}$ H. Linnartz, J.-B. Bossa, J. Bouwman, H. M. Cuppen, S. H. Cuylle, E. F. van Dishoeck, E. C. Fayolle, G. Fedoseev, G. W. Fuchs, S. Ioppolo, K. Isokoski, T. Lamberts, K. I. Öberg, C. Romanzin, E. Tenenbaum, and J. Zhen, in Proceedings IAU Symposium No. 280 "The Molecular Universe," edited by José Cernicharo and Rafael Bachiller (Cambridge University Press, 2011), p. 390.

${ }^{19}$ P. Theulé, F. Duvernay, G. Danger, F. Borget, J.-B. Bossa, V. Vinogradoff, F. Mispelaer, and T. Chiavassa, Adv. Space Res. 52, 1567 (2013).

${ }^{20} \mathrm{~K}$. Isokoski, "Physics and chemistry of interstellar ice," Ph.D. thesis (Leiden Observatory, Leiden University, 2013).

${ }^{21}$ K. Isokoski, J.-B. Bossa, T. Triemstra, and H. Linnartz, Phys. Chem. Chem. Phys. 16, 3456 (2014).

${ }^{22}$ B. M. Jones and R. I. Kaiser, J. Phys. Chem. Lett. 4, 1965 (2013).

${ }^{23}$ R. I. Kaiser, S. Maity, and B. M. Jones, Phys. Chem. Chem. Phys. 16, 3399 (2014).

${ }^{24}$ M. S. Gudipati and R. Yang, Astrophys. J. Lett. 756, L24 (2012).

${ }^{25}$ R. Yang and M. S. Gudipati, J. Chem. Phys. 140, 104202 (2014).

${ }^{26}$ M. E. Belov, S. S. Alimpiev, V. V. Mlynsky, S. M. Nikiforov, and P. J. Derrick, Rapid Commun. Mass Spectrom. 9, 1431 (1995).

${ }^{27}$ S. Berkenkamp, M. Karas, and F. Hillenkamp, Proc. Natl. Acad. Sci. U.S.A. 93, 7003 (1996).

${ }^{28}$ O. Ellegaard and J. Schou, J. Appl. Phys. 83, 1078 (1998).

${ }^{29}$ S. Georgiou and A. Koubenakis, Chem. Rev. 103, 349 (2003).

${ }^{30}$ C. Mihesan, M. Ziskind, B. Chazallon, E. Therssen, P. Desgroux, and C. Focsa, Surf. Sci. 593, 221 (2005).

${ }^{31}$ C. Mihesan, M. Ziskind, B. Chazallon, C. Focsa, and J. Destombes, Appl. Surf. Sci. 248, 238 (2005)

${ }^{32}$ A. Pirkl, J. Soltwisch, F. Draude, and K. Dreisewerd, Anal. Chem. 84, 5669 (2012).

${ }^{33}$ R. Nelson, M. Rainbow, D. Lohr, and P. Williams, Science 246, 1585 (1989).

${ }^{34}$ D. Schieltz, C.-W. Chou, C.-W. Luo, R. Thomas, P. Williams, and C. Becker, Rapid Commun. Mass Spectrom. 6, 631 (1992).

${ }^{35}$ F. Focsa and J. Destombes, Chem. Phys. Lett. 347, 390 (2001).

${ }^{36}$ A. Ikeda, M. Matsumoto, S. Ogura, T. Okano, and K. Fukutani, J. Chem. Phys. 138, 124705 (2013).
${ }^{37}$ K. I. Öberg, G. W. Fuchs, Z. Awad, H. J. Fraser, S. Schlemmer, E. F. van Dishoeck, and H. Linnartz, Astrophys. J. Lett. 662, L23 (2007).

${ }^{38}$ J. H. Lacy, J. S. Carr, N. J. Evans, F. Baas, J. M. Achtermann, and J. F. Arens, Astrophys. J. 376, 556 (1991).

${ }^{39}$ A. C. A. Boogert, W. A. Schutte, A. G. G. M. Tielens, D. C. B. Whittet, F. P. Helmich, P. Ehrenfreund, P. R. Wesselius, T. de Graauw, and T. Prusti, Astron. Astrophys. 315, L377 (1996).

${ }^{40}$ E. L. Gibb, D. C. B. Whittet, A. C. A. Boogert, and A. G. G. M. Tielens, Astrophys. J. Supp. 151, 35 (2004).

${ }^{41}$ K. I. Öberg, A. C. A. Boogert, K. M. Pontoppidan, G. A. Blake, N. J. Evans, F. Lahuis, and E. F. van Dishoeck, Astrophys. J. 678, 1032 (2008).

${ }^{42}$ Y.-J. Chen, K.-J. Chuang, G. M. Muñoz Caro, M. Nuevo, C.-C. Chu, T.-S. Yih, W.-H. Ip, and C.-Y. R. Wu, Astrophys. J. 781, 15 (2014).

${ }^{43}$ J.-B. Bossa, K. Isokoski, M. S. de Valois, and H. Linnartz, Astron. Astrophys. 545, A82 (2012).

${ }^{44}$ D. M. Hudgins, S. A. Sandford, L. J. Allamandola, and A. G. G. M. Tielens, Astrophys. J., Suppl. Ser. 86, 713 (1993).

${ }^{45}$ M. S. Westley, G. A. Baratta, and R. A. Baragiola, J. Chem. Phys. 108, 3321 (1998)

${ }^{46}$ S. E. Stein, "Mass spectra" NIST Chemistry WebBook, NIST Standard Reference Database Number 69, edited by P. J. Linstrom and W. G. Mallard (National Institute of Standards and Technology, Gaithersburg, 2014), see http://webbook.nist.gov (retrieved May 7, 2014).

${ }^{47}$ K. Håkansson, R. Zubarev, and P. Håkansson, Rapid Commun. Mass Spectrom. 12, 705 (1998).

${ }^{48}$ A. C. Sinnock and B. L. Smith, Phys. Lett. A 28, 22 (1968).

${ }^{49}$ S. S. Prasad and S. P. Tarafdar, Astrophys. J. 267, 603 (1983).

${ }^{50}$ Y.-J. Wu, C. Y. R. Wu, S.-L. Chou, M.-Y. Lin, H.-C. Lu, J.-I. Lo, and B.-M. Cheng, Astrophys. J. 746, 175 (2012).

${ }^{51}$ P. A. Gerakines, W. A. Schutte, and P. Ehrenfreund, Astron. Astrophys. 312, 289 (1996)

${ }^{52}$ G. A. Baratta, G. Leto, and M. E. Palumbo, Astron. Astrophys. 384, 343 (2002).

${ }^{53}$ C. J. Bennett, C. S. Jamieson, Y. Osamura, and R. I. Kaiser, Astrophys. J. 653, 792 (2006)

${ }^{54}$ J. Lagarias, J. Reeds, M. Wright, and P. Wright, SIAM. J. Optim. 9, 112 (1998).

${ }^{55}$ Y.-K. Kim, K. K. Irikura, M. E. Rudd, M. A. Ali, P. M. Stone, J. Chang, J. S. Coursey, R. A. Dragoset, A. R. Kishore, K. J. Olsen, A. M. Sansonetti, G. G. Wiersma, D. S. Zucker, and M. A. Zucker, "Electron-impact cross sections for ionization and excitation," NIST Standard Reference Database Number 107, see http://www.nist.gov/pml/data/ionization/ (retrieved September 20, 2014). 